\title{
CHARACTERIZATIONS OF RICCI FLAT METRICS AND LAGRANGIAN SUBMANIFOLDS IN TERMS OF THE VARIATIONAL PROBLEM
}

\author{
TETSUYA TANIGUCHI \\ Department of Mathematics, School of General Education, \\ Kitasato University, \\ Sagamihara, Kanagawa 228-8555, Japan \\ e-mail: tetsuya@kitasato-u.ac.jp \\ and SEIICHI UDAGAWA \\ Division of Mathematics, School of Medicine, \\ Nihon University, Itabashi, Tokyo 173-0032, Japan \\ e-mail: udagawa.seiichi@nihon-u.ac.jp \\ Dedicated to Professor Kazumi Tsukada for his sixtieth birthday
}

(Received 21 November 2013; revised 12 February 2014; accepted 8 April 2014; first published online 17 December 2014)

\begin{abstract}
Given the pair $(P, \eta)$ of $(0,2)$ tensors, where $\eta$ defines a volume element, we consider a new variational problem varying $\eta$ only. We then have Einstein metrics and slant immersions as critical points of the 1st variation. We may characterize Ricci flat metrics and Lagrangian submanifolds as stable critical points of our variational problem.
\end{abstract}

2010 Mathematics Subject Classification. Primary 53D12, 53C25; Secondary 53C42, 49Q99.

1. Introduction. The variational problems to obtain typical geometric objects, such as minimal surfaces, harmonic mappings, Einstein metrics and Yang-Mills connections, are the central topics in Riemannian geometry. The important geometric structure for the geometric objects is the curvature tensor of the metric. However, since the curvature tensor itself is too complicated and difficult to treat, one may consider the Ricci tensor, denoted by Ric, which is easier to treat than the curvature tensor. In 1916, Albert Einstein used the Ricci tensor to describe the gravitational field equation. When the spacetime is empty and the cosmological constant is zero, Einstein's field equation becomes Ric $-\frac{S}{2} g=0$ [2], where $g$ is the Lorentzian metric and $S$ is the trace of Ric with respect to $g$ called a scalar curvature, and consequently Ric $=0$ holds. A Riemannian or Lorentzian metric satisfying the latter condition is called a Ricci flat metric. The existence of such a Ricci flat metric is ensured for a compact Kähler manifold with zero 1st Chern class because of the solution for the Calabi conjecture by S. T. Yau [5], which led to the concept of the Calabi-Yau manifold in mirror symmetry. More generally, a Riemannian or Lorentzian metric $g$ satisfying Ric $=\frac{S}{n} g$ is called an Einstein metric, where $n$ is the dimension of the manifold. It is known that Einstein metric is a solution for the variational problem of the function of the total scalar curvature divided by the $\frac{n-2}{n}$-power of the volume of the Riemannian manifold (we 
consider only the case of compact Riemannian manifold). In mirror symmetry, the Lagrangian submanifolds of the symplectic manifold play an important role. We then ask the question "Is the Lagrangian submanifold the solution of certain variational problem?".

In this paper, we introduce a new variational problem on the space $\mathfrak{T}_{2}^{0} \times \mathfrak{T}_{2}^{0 *}$ of the tensor product of the bundle of smooth $(0,2)$-tensors and the bundle of smooth $(0,2)$ tensors with non-zero determinant. Given a $(P, \eta) \in \mathfrak{T}_{2}^{0} \times \mathfrak{T}_{2}^{0 *}$, fixing $P$ we consider the function $E(\eta)$ (see Section 1). Varying the tensor $\eta$, we calculate the 1 st and 2nd variation formulae for the variational problem. Consequently, the critical point of the 1 st variation is given by the equation $P=\frac{2 \mathfrak{p}}{n} \eta$. From this, we obtain an Einstein metric or a slant immersion as the critical point when $P$ is the Ricci tensor or $f^{*} \Omega_{N}$, respectively (for details, see Corollaries in Section 4). For the stability problem of the critical points, we prove that if $n \geqq 3$ and $\mathfrak{p}$ is a non-zero, then the critical point of $E(\eta)$ is unstable (Theorem 5.5). As corollaries of this theorem, we may characterize the Ricci flat metric or a Lagrangian immersion as the stable critical point of $E(\eta)$, where we take $\eta$ to be a Riemannian metric or a symplectic form, respectively (see Corollaries in Section 5).

2. New variational problem. Let $(M, g)$ be an $n$-dimensional Riemannian manifold. Choose a local coordinate system $\left\{x^{1}, x^{2}, \ldots x^{n}\right\}$ of $M$. Then, the Riemannian metric $g$ is expressed as $g=\sum_{i, j=1}^{n} g_{i j} d x^{i} d x^{j}$. The standard measure $d \mu_{g}$ of $(M, g)$ is given by

$$
d \mu_{g}=\sqrt{\operatorname{det}\left(g_{\ddot{j}}\right)} d x^{1} d x^{2} \ldots d x^{n} .
$$

We denote by $R$, Ric and $S$ the curvature tensor, the Ricci tensor and the scalar curvature of $(M, g)$, respectively. A famous variational problem in Riemannian geometry is to extremise the function

$$
J(g)=\frac{\int_{M} S d \mu_{g}}{\left(\int_{M} d \mu_{g}\right)^{\frac{n-2}{n}}}
$$

The critical point of $J(g)$ is a metric $g$, which satisfies $R i c=\frac{S}{n} g$, that is, $g$ is an Einstein metric.

We now introduce a new variational problem. Let $M$ be an $n$-dimensional smooth manifold. We assume that, in this paper, $M$ is connected. Let $\mathfrak{T}_{2}^{0}=\mathfrak{T}_{2}^{0}(M)$ be the set of all smooth $(0,2)$-tensors on $M$. Denote by $\mathfrak{T}_{2}^{0 *}=\mathfrak{T}_{2}^{0 *}(M)$ the subset of all smooth $(0,2)$-tensors $\eta^{\prime}$ s with $\operatorname{det} \eta \neq 0$. Choose a local coordinate system $\left\{x^{1}, x^{2}, \ldots x^{n}\right\}$ of $M$. Given an $\eta=\sum_{i, j=1}^{n} \eta_{i j} d x^{i} \otimes d x^{j} \in \mathfrak{T}_{2}^{0 *}$, we define a $d \mu_{\eta}$ by

$$
d \mu_{\eta}=\sqrt{\left|\operatorname{det}\left(\eta_{i j}\right)\right|} d x^{1} d x^{2} \ldots d x^{n} .
$$

We then define a function $I$ on $\mathfrak{T}_{2}^{0} \times \mathfrak{T}_{2}^{0 *}$ by

$$
I(P, \eta)=\int_{M} \operatorname{trace}_{\eta} P d \mu_{\eta} \quad \text { for }(P, \eta) \in \mathfrak{T}_{2}^{0} \times \mathfrak{T}_{2}^{0 *},
$$


where $\operatorname{trace}_{\eta} P=\sum_{i, j=1}^{m} P_{i j} \eta^{j i}$ and $\eta^{i j}$ is the component of the inverse matrix of the matrix $\left(\eta_{i j}\right)$. We normalize $I$ so that it is invariant under the homothetic transformation $\eta \mapsto \lambda \eta$. Next, fixing $P \in \mathfrak{T}_{2}^{0}$, we define $E(\eta)$ by

$$
E(\eta):=\frac{\int_{M} \operatorname{trace}_{\eta} P d \mu_{\eta}}{\left(\int_{M} d \mu_{\eta}\right)^{\frac{n-2}{n}}} .
$$

For applications, we mainly consider the case where $\eta$ is a symmetric tensor or a skew-symmetric tensor.

3. Slant immersion. Let $(M, \Omega)$ be a $2 m$-dimensional symplectic manifold, that is, $\Omega$ is a closed real nondegenerate 2-form. It is known that $M$ admits an almost Kähler structure $(g, J)$ with $\Omega(X, Y)=g(J X, Y)$ (cf. [4]). Let $\left(M, \Omega_{M}\right)$ and $\left(N, \Omega_{N}\right)$ be symplectic manifolds. We may give almost Kähler structures $\left(g_{M}, J_{M}\right)$ and $\left(g_{N}, J_{N}\right)$ on $M$ and $N$ in the standard way, respectively. Consider an isometric immersion $\varphi:\left(M, g_{M}\right) \longrightarrow\left(N, g_{N}\right)$. For any $X \in T_{x} M, x \in M, \varphi_{*}(X)$ is a vector in $\varphi_{*}\left(T_{x} M\right) \subset$ $T_{\varphi(x)} N$. However, $J_{N} \varphi_{*}(X)$ is not necessarily in $\varphi_{*}\left(T_{x} M\right)$ but in $T_{\varphi(x)} N$. With respect to the orthogonal direct sum decomposition $T_{\varphi(x)} N=\varphi_{*}\left(T_{x} M\right)+N_{x}$, denote by $\pi_{x}$ : $T_{\varphi(x)} N \longrightarrow \varphi_{*}\left(T_{x} M\right)$ the orthogonal projection along $M$. We denote by $\theta_{X}$ the angle between $J_{N} \varphi_{*}(X)$ and $\varphi_{*}\left(T_{x} M\right)$. For $X$ of unit length, we have

$$
\cos \theta_{X}=\frac{\left\langle J_{N} \varphi_{*}(X), \pi\left(J_{N} \varphi_{*}(X)\right)\right\rangle}{\left|\pi\left(J_{N} \varphi_{*}(X)\right)\right|} .
$$

Since $\left|\pi\left(J_{N} \varphi_{*}(X)\right)\right|=\cos \theta_{X}$, we obtain the following.

$$
\cos ^{2} \theta_{X}=\left\langle J_{N} \varphi_{*}(X), \pi\left(J_{N} \varphi_{*}(X)\right)\right\rangle=\sum_{i=1}^{2 m}\left\langle J_{N} \varphi_{*}(X), \varphi_{*}\left(\mathbf{e}_{i}\right)\right\rangle^{2},
$$

where $\left\{\mathbf{e}_{1}, \mathbf{e}_{2}, \ldots, \mathbf{e}_{2 m}\right\}$ is an orthonormal basis of $T_{x} M$.

Definition. An isometric immersion $\varphi:\left(M, g_{M}\right) \longrightarrow\left(N, g_{N}, J_{N}\right)$ is called slant if $\theta_{X}$ is constant for any non-zero $X$. When $\varphi$ is slant, the angle $\theta=\theta_{X}$ for non-zero $X$ is called slant angle.

Lemma 3.1 (cf. [3]). Let $K$ be a non-negative real number. Then, $\varphi$ is slant with slant angle $\cos ^{-1}(K)$ if and only if

$$
\sum_{k=1}^{2 m}\left\langle J_{N} \varphi_{*}\left(\mathbf{e}_{i}\right), \varphi_{*}\left(\mathbf{e}_{k}\right)\right\rangle\left\langle J_{N} \varphi_{*}\left(\mathbf{e}_{j}\right), \varphi_{*}\left(\mathbf{e}_{k}\right)\right\rangle=K^{2} \delta_{i j}
$$

for $i, j=1,2, \ldots, 2 m$.

REMARK. If $\varphi^{*} \Omega_{N}=K \Omega_{M}$ for some real number $K$, then it follows from Lemma 3.1 that $\varphi$ is slant with slant angle $\cos ^{-1}(|K|)$. 
4. 1st variation formulae. For the variation $\left\{\eta_{t}\right\}$ of $\eta \in \mathfrak{T}_{2}^{0 *}$ with $\eta_{0}=\eta$, we denote by $\delta \eta$ the section $\left.\frac{d}{d t}\right|_{t=0} \eta_{t} \in \mathfrak{T}_{2}^{0}$. Assume that $M$ is an $n$-dimensional compact manifold with an $\eta \in \mathfrak{T}_{2}^{0 *}$. Take $P \in \mathfrak{T}_{2}^{0}$. Define $V, \mathfrak{p}, U$ and $c$ by

$$
V=\int_{M} d \mu_{\eta}, \quad \mathfrak{p}=\frac{1}{2} \sum_{i, j=1}^{n} P_{\ddot{j}} \eta^{j i}, \quad U=\int_{M} \mathfrak{p} d \mu_{\eta}, \quad c=\frac{n-2}{n} .
$$

We then see that $E(\eta)=2 U / V^{c}$. Fixing $\eta \in \mathfrak{T}_{2}^{0 *}$, we now introduce the inner products $\langle$,$\rangle and \langle\langle\rangle$,$\rangle on \mathfrak{T}_{2}^{0}$ by

$$
\begin{aligned}
\langle p, q\rangle & =\sum_{i, j, k, l=1}^{n} p_{i j} \eta^{j k} q_{k l} \eta^{l i}, \\
\langle\langle p, q\rangle\rangle & =\int_{M}\langle p, q\rangle d \mu_{\eta} \quad \text { for } \quad p, q \in \mathfrak{T}_{2}^{0} .
\end{aligned}
$$

Using the relation $\sum_{j=1}^{n} \eta_{i j} \eta^{j k}=\delta_{i}^{k}$, we have the following fundamental fact.

LEMMA 4.1.

$$
\left\{\begin{array}{l}
\delta \eta^{i j}=-\sum_{k, l=1}^{n} \eta^{i k} \delta \eta_{k l} \eta^{l j} \\
\delta\left(d \mu_{\eta}\right)=\frac{1}{2} \sum_{i, j=1}^{n} \eta^{\ddot{j}} \delta \eta_{j i} d \mu_{\eta}
\end{array}\right.
$$

Using Lemma 4.1 we obtain the following.

LEMMA 4.2.

$$
\left\{\begin{array}{l}
\delta \mathfrak{p}=-\frac{1}{2}\langle P, \delta \eta\rangle, \\
\delta V=\frac{1}{2}\langle\langle\eta, \delta \eta\rangle\rangle, \\
\delta U=\frac{1}{2}\langle\langle\mathfrak{p} \eta-P, \delta \eta\rangle\rangle .
\end{array}\right.
$$

Using Lemma 4.2, we now calculate the 1st variation of the function $E(\eta)$ fixing $P \in \mathfrak{T}_{2}^{0}$.

$$
\begin{aligned}
\delta E(\eta) & =\frac{2 \delta U \cdot V^{c}-2 U \cdot \delta V^{c}}{V^{2 c}} \\
& =\frac{1}{V^{2 c}}\left(\langle\langle\mathfrak{p} \eta-P, \delta \eta\rangle\rangle V^{c}-c U V^{c-1}\langle\langle\eta, \delta \eta\rangle\rangle\right) \\
& =\frac{1}{V^{c+1}}\langle\langle V(\mathfrak{p} \eta-P)-c U \eta, \delta \eta\rangle\rangle .
\end{aligned}
$$

Consequently, we obtain the following formulae.

PROPOSITION 4.3. $\delta E(\eta)=0$ if and only if $P=\left(\mathfrak{p}-c \frac{U}{V}\right) \eta=\frac{2 \mathfrak{p}}{n} \eta$, where $\mathfrak{p}$ is equal to a constant $\frac{U}{V}$ for $n \neq 2$. 
The unproved assertion in Proposition 4.3 follows from multiplying $P=\left(\mathfrak{p}-c \frac{U}{V}\right) \eta$ by $\eta^{-1}$ and taking the trace of the resulting equation.

Corollary 4.4. Consider the case where $P$ is the Ricci tensor of a Riemannian manifold $(M, g)$, and take $\eta=g$. Then, $\delta E(g)=0$ if and only if $g$ is an Einstein metric.

Corollary 4.5. Consider the case where $P$ is the Ricci form of an almost Kähler manifold $\left(M, g_{M}, J_{M}, \Omega_{M}\right)$, that is, $P(X, Y)=\operatorname{Ric}\left(J_{M} X, Y\right)$, and take $\eta=\Omega_{M}$. Then, $\delta E\left(\Omega_{M}\right)=0$ if and only if $g_{M}$ is an Einstein metric.

Corollary 4.6. Let $\varphi:\left(M, g_{M}, J_{M}, \Omega_{M}\right) \longrightarrow\left(N, g_{N}, J_{N}, \Omega_{N}\right)$ be an isometric immersion from a $2 m$-dimensional compact almost Kähler manifold into an almost Kähler manifold. Consider the case where $P=\varphi^{*} \Omega_{N}$ and $m \geqq 2$. If $\delta E\left(\Omega_{M}\right)=0$ then $\varphi$ is slant with slant angle $\cos ^{-1}\left(\frac{U}{m V}\right)$.

In these corollaries, we considered the variations of $\eta$ in the space of symmetric tensors or skew-symmetric tensors accordingly as $P$ is symmetric or skew-symmetric. However, since the local minimum of $E(\eta)$ is not always zero, it is possible to consider the variation of $\eta$ in the other direction than $P$.

COROLlary 4.7. Consider the case where $P$ is the Ricci tensor of an almost Kähler manifold $\left(M, g_{M}, J_{M}, \Omega_{M}\right)$. Then, $\delta E\left(\Omega_{M}\right)=0$ if and only if $g_{M}$ is a Ricci flat metric.

COROLlary 4.8. Let $\left(N, \Omega_{N}\right)$ be a symplectic manifold and $(M, g)$ be a Riemannian manifold with $\operatorname{dim} M=\frac{1}{2} \operatorname{dim} N$. Let $\varphi: M \longrightarrow N$ be an immersion. Consider the case where $P=\varphi^{*} \Omega_{N}$ and $\eta=g$. Then, $\delta E(g)=0$ if and only if $\varphi$ is a Lagrangian immersion.

5. 2nd variation formulae. We calculate the second variation of $E(\eta)$ under the condition that $\eta$ is an element of $\mathfrak{T}_{2}^{0 *}$ with $\delta E(\eta)=0$. Set

$$
H=V(\mathfrak{p} \eta-P)-c U \eta .
$$

Then, since $\delta E(\eta)=\frac{1}{V^{c+1}}\langle\langle H, \delta \eta\rangle\rangle$ by Proposition 4.3, we see that $\delta E(\eta)=0$ if and only if $H=0$. Therefore, we obtain the following.

$$
\delta^{2} E(\eta)=\frac{1}{V^{c+1}}\langle\langle\delta H, \delta \eta\rangle\rangle .
$$

We use the notation that $\langle\langle f\rangle\rangle=\int_{M} f d \mu_{g}$ for any smooth function on $M$. We then may write

$$
\langle\langle\eta, \delta \eta\rangle\rangle=\int_{M} \operatorname{trace}_{\eta} \delta \eta d \mu_{\eta}=\left\langle\left\langle\operatorname{trace}_{\eta} \delta \eta\right\rangle\right\rangle, \quad U=\int_{M} \mathfrak{p} d \mu_{\eta}=\langle\langle\mathfrak{p}\rangle\rangle .
$$

Now, it is enough to calculate $\delta H$. From Lemma 4.2, we calculate

$$
\begin{aligned}
\delta H= & \delta V \cdot(\mathfrak{p} \eta-P)+V \cdot \delta \mathfrak{p} \cdot \eta+V \mathfrak{p} \cdot \delta \eta \\
& -c \delta U \cdot \eta-c U \cdot \delta \eta \\
= & \frac{1}{2}\langle\langle\eta, \delta \eta\rangle\rangle(\mathfrak{p} \eta-P)-\frac{1}{2} V\langle P, \delta \eta\rangle \eta+V \mathfrak{p} \delta \eta \\
& -\frac{c}{2}\langle\langle\mathfrak{p} \eta-P, \delta \eta\rangle\rangle \eta-c U \delta \eta .
\end{aligned}
$$


Thus, it follows that

$$
\begin{aligned}
V^{c+1} \delta^{2} E(\eta)= & \frac{1}{2}\left\langle\left\langle\operatorname{trace}_{\eta} \delta \eta\right\rangle\right\rangle\langle\langle\mathfrak{p} \eta-P, \delta \eta\rangle\rangle-\frac{1}{2} V\left\langle\left\langle\left(\operatorname{trace}_{\eta} \delta \eta\right) P, \delta \eta\right\rangle\right\rangle \\
& +V\langle\langle\mathfrak{p} \delta \eta, \delta \eta\rangle\rangle-\frac{c}{2}\left\langle\left\langle\operatorname{trace}_{\eta} \delta \eta\right\rangle\right\rangle\langle\langle\mathfrak{p} \eta-P, \delta \eta\rangle\rangle-c U\langle\langle\delta \eta, \delta \eta\rangle\rangle \\
= & \frac{1-c}{2}\left\langle\left\langle\operatorname{trace}_{\eta} \delta \eta\right\rangle\right\rangle\langle\langle\mathfrak{p} \eta-P, \delta \eta\rangle\rangle-\frac{1}{2} V\left\langle\left\langle\left(\operatorname{trace}_{\eta} \delta \eta\right) P, \delta \eta\right\rangle\right\rangle \\
& +V\langle\langle\mathfrak{p} \delta \eta, \delta \eta\rangle\rangle-c U\langle\langle\delta \eta, \delta \eta\rangle\rangle
\end{aligned}
$$

We now consider the case $n \neq 2$. In this case, $\mathfrak{p}$ is a constant and $P=\frac{2 \mathfrak{p}}{n} \eta$ holds. We hence obtain the following.

$$
\begin{aligned}
V^{c+1} \delta^{2} E(g)= & \frac{(n-2) \mathfrak{p}}{n^{2}}\left\langle\left\langle\operatorname{trace}_{\eta} \delta \eta\right\rangle\right\rangle^{2} \\
& -\frac{\mathfrak{p} V}{n}\left\langle\left\langle\left(\operatorname{trace}_{\eta} \delta \eta\right)^{2}\right\rangle\right\rangle+\frac{2 \mathfrak{p} V}{n}\langle\langle\delta \eta, \delta \eta\rangle\rangle .
\end{aligned}
$$

Next, consider the case $n=2$. In this case, we have $c=0$ and $P=\mathfrak{p} \eta$. Therefore, we obtain the following.

$$
V^{c+1} \delta^{2} E(\eta)=-\frac{V}{2}\left\langle\left\langle\mathfrak{p}\left(\operatorname{trace}_{\eta} \delta \eta\right)^{2}\right\rangle\right\rangle+V\langle\langle\mathfrak{p} \delta \eta, \delta \eta\rangle\rangle .
$$

Consequently, we conclude the following.

THEOREM 5.4. Fixing $P$, under the condition that $\delta E(\eta)=0$ the 2 nd variation formulae is given by

$$
\begin{aligned}
& \delta^{2} E(\eta) \\
& =\frac{1}{V^{c+1}}\left\{\frac{n-2}{n^{2} V}\langle\langle\mathfrak{p}\rangle\rangle\left\langle\left\langle\operatorname{trace}_{\eta} \delta \eta\right\rangle\right\rangle^{2}-\frac{V}{n}\left\langle\left\langle\mathfrak{p}\left(\operatorname{trace}_{\eta} \delta \eta\right)^{2}\right\rangle\right\rangle+\frac{2 V}{n}\langle\langle\mathfrak{p} \delta \eta, \delta \eta\rangle\rangle\right\},
\end{aligned}
$$

where $\mathfrak{p}$ is a constant when $n \neq 2$. In particular, if $n=1,2$ and $\mathfrak{p}$ is a non-negative(resp. non-positive) constant then $\delta^{2} E(\eta) \geqq 0$ (resp. $\left.\delta^{2} E(\eta) \leqq 0\right)$ holds.

The last assertion in Theorem 5.4 follows from the Cauchy-Schwarz inequality

$$
\left\langle\left\langle\left(\operatorname{trace}_{\eta} \delta \eta\right)^{2}\right\rangle\right\rangle \leqq n\langle\langle\delta \eta, \delta \eta\rangle\rangle .
$$

When $n \geqq 3$ we have the following.

THEOREM 5.5. Assume that $n \geqq 3$ and $\mathfrak{p}$ is a non-zero. Then, the critical point of $E(\eta)$ is unstable.

Proof. Note that $\mathfrak{p}$ is a constant when $n \geqq 3$. First of all, consider the case where $\mathfrak{p}$ is a negative constant. In this case, if we choose $\delta \eta$ so that trace $\delta \delta \eta=0$ holds, then we see that $\delta^{2} E(\eta)<0$. In the following, we consider the case where $\mathfrak{p}$ is a positive constant. Choose two domains $V_{1}, V_{2}$ in $M$ with $V_{1} \cap V_{2}=\phi$. Choose $\epsilon>0$ so that

$$
\epsilon<\frac{n-2}{n} \cdot \operatorname{Vol}\left(V_{1}\right) \text { and } \epsilon<\frac{n-2}{n} \cdot \operatorname{Vol}\left(V_{2}\right)
$$


hold. Next, we choose two domains $U_{1}, U_{2}$ in $M$ so that they satisfy

$$
\left\{\begin{array}{llll}
V_{1} \supset U_{1}, & & \operatorname{Vol}\left(V_{1}\right)-\operatorname{Vol}\left(U_{1}\right)<\epsilon \\
V_{2} \supset U_{2}, & & \operatorname{Vol}\left(V_{2}\right)-\operatorname{Vol}\left(U_{2}\right)<\epsilon
\end{array}\right.
$$

We define a $\widetilde{f} \in C^{\infty}(M)$ by

$$
\widetilde{f}= \begin{cases}1 & \text { on } U_{1} \\ \geqq 0 & \text { on } V_{1} \\ \leqq 0 & \text { on } V_{2} \\ -1 & \text { on } U_{2} \\ 0 & \text { Others. }\end{cases}
$$

Set $c_{1}=\int_{V_{1}} \tilde{f} d \mu_{\eta}, c_{2}=-\int_{V_{2}} \tilde{f} d \mu_{\eta}$. Then, we see that $c_{1}>0$ and $c_{2}>0$. Moreover, if we define $f$ by

$$
f= \begin{cases}c_{2} \widetilde{f} & \text { on } V_{1} \\ c_{1} \widetilde{f} & \text { on } V_{2} \\ 0 & \text { Others }\end{cases}
$$

then we have $f \in C^{\infty}(M)$. We consider $\delta \eta=f \eta$. We calculate

$$
\left\langle\left\langle\operatorname{trace}_{\eta} \delta \eta\right\rangle\right\rangle=\int_{V_{1}} n c_{2} \tilde{f} d \mu_{\eta}+\int_{V_{2}} n c_{1} \tilde{f} d \mu_{\eta}=n c_{2} c_{1}+n c_{1}\left(-c_{2}\right)=0
$$

In particular, we see that the term $\left\langle\left\langle\operatorname{trace}_{\eta} \delta \eta\right\rangle\right\rangle^{2}$ is equal to zero. Moreover, using the definition of the function $f$, we have the following.

$$
\begin{aligned}
-\frac{\mathfrak{p} V}{n}\left\langle\left\langle\left(\operatorname{trace}_{\eta} \delta \eta\right)^{2}\right\rangle\right\rangle & =-\frac{\mathfrak{p} V}{n} \int_{V_{1}} f^{2} n^{2} d \mu_{\eta}-\frac{\mathfrak{p} V}{n} \int_{V_{2}} f^{2} n^{2} d \mu_{\eta} \\
& \leqq-\frac{\mathfrak{p} V}{n} \int_{U_{1}} c_{2}^{2} n^{2} d \mu_{\eta}-\frac{\mathfrak{p} V}{n} \int_{U_{2}} c_{1}^{2} n^{2} d \mu_{\eta} \\
& =-n \mathfrak{p} V\left\{c_{2}^{2} \operatorname{Vol}\left(U_{1}\right)+c_{1}^{2} \operatorname{Vol}\left(U_{2}\right)\right\}, \\
\frac{2 \mathfrak{p} V}{n}\langle\langle\delta \eta, \delta \eta\rangle\rangle & =\frac{2 \mathfrak{p} V}{n}\left\{\int_{V_{1}} n f^{2} d \mu_{\eta}+\int_{V_{2}} n f^{2} d \mu_{\eta}\right\} \\
& \leqq \frac{2 \mathfrak{p} V}{n}\left\{\int_{V_{1}} n c_{2}^{2} d \mu_{\eta}+\int_{V_{2}} n c_{1}^{2} d \mu_{\eta}\right\} \\
& =2 \mathfrak{p} V\left\{c_{2}^{2} \operatorname{Vol}\left(V_{1}\right)+c_{1}^{2} \operatorname{Vol}\left(V_{2}\right)\right\} .
\end{aligned}
$$


It then follows from (5.6)-(5.9) and the assumption $n \geqq 3$ that

$$
\begin{aligned}
\delta^{2} E(\eta) & =\frac{1}{V^{c+1}}\left[0-\frac{\mathfrak{p} V}{n}\left\langle\left\langle\left(\operatorname{trace}_{\eta} \delta \eta\right)^{2}\right\rangle\right\rangle+\frac{2 \mathfrak{p} V}{n}\langle\langle\delta \eta, \delta \eta\rangle\rangle\right] \\
& \leqq \frac{1}{V^{c+1}}\left[-\mathfrak{p} \operatorname{Vn}\left\{c_{2}^{2} \operatorname{Vol}\left(U_{1}\right)+c_{1}^{2} \operatorname{Vol}\left(U_{2}\right)\right\}+2 \mathfrak{p} V\left\{c_{2}^{2} \operatorname{Vol}\left(V_{1}\right)+c_{1}^{2} \operatorname{Vol}\left(V_{2}\right)\right\}\right] \\
& <\frac{\mathfrak{p} V}{V^{c+1}}\left[c_{2}^{2}\left\{2 \operatorname{Vol}\left(V_{1}\right)+n\left(-\operatorname{Vol}\left(V_{1}\right)+\epsilon\right)\right\}+c_{1}^{2}\left\{2 \operatorname{Vol}\left(V_{2}\right)+n\left(-\operatorname{Vol}\left(V_{2}\right)+\epsilon\right)\right\}\right] \\
& =\frac{n \mathfrak{p}}{V^{c}}\left[c_{2}^{2}\left\{-\frac{n-2}{n} \operatorname{Vol}\left(V_{1}\right)+\epsilon\right\}+c_{1}^{2}\left\{-\frac{n-2}{n} \operatorname{Vol}\left(V_{2}\right)+\epsilon\right\}\right]<0
\end{aligned}
$$

Therefore, the critical point of $E(\eta)$ is unstable.

Theorem 5.5, together with Proposition 4.3, yields the following.

THEOREM 5.10. Let $(M, g)$ be a smooth manifold. For $E: \mathfrak{T}_{2}^{0 *} \longrightarrow \mathbf{R}$ defined in Section 1, the following four conditions are mutually equivalent.

(1) There exists a critical point $\eta \in \mathfrak{T}_{2}^{0 *}$ such that $\operatorname{trace}_{\eta} P=0$.

(2) $\eta$ is a critical point for any $\eta \in \mathfrak{T}_{2}^{0 *}$.

(3) $\eta$ is a stable critical point for any $\eta \in \mathfrak{T}_{2}^{0 *}$.

(4) $P=0$.

Moreover, if $\operatorname{dim} M \geqq 3$ then any of the following conditions (5) and (6) is also equivalent to any of the conditions (1), (2), (3) and (4)

(5) There exist a critical point $\eta \in \mathfrak{T}_{2}^{*}$ and a point $m \in M$ such that $\operatorname{trace}_{\eta} P(m)=0$ or $\operatorname{det}\left(P_{i j}\right)(m)=0$.

(6) There exists a stable critical point $\eta \in \mathfrak{T}_{2}^{0 *}$.

Proof. It is enough to prove the implication (2) $\Longrightarrow(1)$. Take any $\eta \in \mathfrak{T}_{2}^{0 *}$. Then, we have $P=\frac{\operatorname{trace}_{\eta} P}{n} \eta$. There are two possibilities : (i) $\operatorname{trace}_{\eta} P \equiv 0$ and (ii) there is a point $m \in M$ such that $\operatorname{trace}_{\eta} P(m) \neq 0$. Since the case (i) implies the condition (1), we just consider the case (ii). In this case, there exists a neighbourhood $U(m)$ of $m \in M$ such that $\operatorname{trace}_{\eta} P \neq 0$ on $U(m)$. Choose $\xi \in \mathfrak{T}_{2}^{0 *}$ so that $\eta$ and $\xi$ are linearly independent on $U(m)$. Set $\eta_{\epsilon}=\eta+\epsilon \xi$. Choosing $\epsilon$ to be small enough, if necessary, we may assume that $\operatorname{trace}_{\eta_{\epsilon}} P \neq 0$ on $U(m)$ and $\eta_{\epsilon} \in \mathfrak{T}_{2}^{0 *}$. We then see that

$$
P=\frac{\operatorname{trace}_{\eta} P}{n} \eta=\frac{\operatorname{trace}_{\eta_{\epsilon}} P}{n} \eta_{\epsilon} \quad \text { on } U(m)
$$

which contradicts the linear independence of $\eta$ and $\xi$.

COROLlaRY 5.11. Consider the case where P is the Ricci tensor of an n-dimensional Riemannian manifold $(M, g), n \geqq 3$ and $\delta E(g)=0$. Then, $g$ is stable if and only if $g$ is a Ricciflat metric.

COROLlaRY 5.12. Consider the case where $P$ is the Ricci form of a $2 m$-dimensional compact almost Kähler manifold $\left(M, g_{M}, J_{M}, \Omega_{M}\right), m \geqq 2$ and $\delta E\left(\Omega_{M}\right)=0$. Then, $\Omega_{M}$ is stable if and only if $g_{M}$ is a Ricci flat metric.

COROLlary 5.13. Let $\varphi:\left(M, g_{M}, J_{M}, \Omega_{M}\right) \longrightarrow\left(N, g_{N}, J_{N}, \Omega_{N}\right)$ be an isometric immersion from a $2 m$-dimensional compact almost Kähler manifold into an almost Kähler manifold. Consider the case where $P=\varphi^{*} \Omega_{N}, m \geqq 2$ and $\delta E\left(\Omega_{M}\right)=0$. Then, $\Omega_{M}$ is 
stable if and only if $\varphi$ is a totally real immersion (i.e., $\varphi^{*} \Omega_{N} \equiv 0[\mathbf{1}]$ ), which, in particular, implies that $\varphi$ is a Lagrangian immersion in case of $\operatorname{dim} N=4 m$.

COROLlARY 5.14. Let $f:(M, g) \longrightarrow(N, h)$ be a smooth map from an m-dimensional semi-Riemannian manifold into an $n$-dimensional manifold with an arbitrary smooth $(0,2)$-tensor $h$. Consider the case where $P=h(d f, d f), m \geqq 3$ and $\delta E(g)=0$. If $g$ is stable or the energy density $e(f)=t r_{g} P / 2$ vanishes at some point, then the energy off is zero, that is

$$
\int_{M} e(f) d \mu_{g}=\int_{M} \frac{1}{2} \sum_{i, j=1}^{m} \sum_{\alpha, \beta=1}^{n} g^{i j} h_{\alpha \beta}(f) f_{i}^{\alpha} f_{j}^{\beta} d \mu_{g}=0 .
$$

In particular, $f$ is a constant map if h is positive or negative definite.

\section{REFERENCES}

1. B.-Y. Chen and K. Ogiue, On totally real submanifolds, Trans. Am. Math. Soc. 193 (1974), 257-266.

2. S. W. Hawking and G. F. Ellis, The large scale structure of space-time, Cambridge Monographs on Mathematical Physics (Cambridge University Press, Cambridge 1973).

3. S. Maeda, Y. Ohnita and S. Udagawa, On slant immersions into Kähler manifolds, Kodai Math. J. 16 (1993), 205-219.

4. D. McDuff and D. Salamon, Introduction to symplectic topology (Oxford University Press, Oxford 1998).

5. S. T. Yau, On the Ricci curvature of a compact Kähler manifold and the complex Monge-Ampere equation I, Commun. Pure Appl. Math. 31 (1978), 339-411. 